\title{
The role of self-peptides in direct T cell allorecognition
}

\author{
Hossam A. Abdelsamed ${ }^{1,2}$ and Fadi G. Lakkis ${ }^{1,3,4}$ \\ 'Thomas E. Starzl Transplantation Institute, Department of Surgery, ${ }^{2}$ Pittsburgh Liver Research Center, ${ }^{3}$ Department of Immunology, and ${ }^{4}$ Department of Medicine, University of Pittsburgh, Pittsburgh, \\ Pennsylvania, USA.
}

\begin{abstract}
Direct allorecognition, the ability of host $\mathrm{T}$ cells to recognize intact allogeneic MHC molecules on transplanted tissues, is often assumed to be less dependent on the peptide bound to the MHC molecule than are other antigen recognition pathways. In this issue of the $J C l$, Son et al. provide unequivocal, in vivo evidence that direct allorecognition depends on the self-peptides bound to the non-self MHC molecule. The authors demonstrate that the induction of allospecific tolerance required the presentation of self-peptides by the non-self MHC molecule, and that only a handful of these peptides accounted for a sizeable proportion of the immunogenicity of the MHC antigen. These are important findings for transplant immunologists because they provide molecular insights into the biology of direct allorecognition, the prime driver of the alloimmune response to MHC-mismatched grafts, and much-needed tools, peptideMHC multimers, to track and study polyclonal alloreactive T cells.
\end{abstract}

\section{Interaction between the TCR} and the peptide-MHC complex

The response of recipient $\mathrm{T}$ cells to nonself antigens present in the transplanted tissue-so-called alloantigens-is the primary predictor of the outcome of solid organ transplantation, whether allograft rejection or tolerance (1). T cell recognition of alloantigens via $\mathrm{T}$ cell receptors (TCRs) is referred to as allorecognition and is mediated by either a direct or an indirect mechanism. Direct allorecognition is predicated on the binding of recipient TCRs to non-self, donor MHC molecules loaded with donor peptides. These peptides are largely derived from ubiquitous, endogenous proteins shared among individuals and are, thus, self to the recipient (Figure 1A) $(2,3)$. On the other hand, indirect allorecognition is mediated by recipient TCRs that recognize nonself donor peptides bound to recipient (self) MHC molecules (Figure 1A). Direct allorecognition accounts for the bulk of an alloimmune response because humans and experimental animals harbor an unusually high frequency of directly alloreactive $\mathrm{T}$ cells, constituting 1\%-10\% of the total $\mathrm{T}$ cell pool for a given $\mathrm{MHC}$ mismatched donor $(4,5)$. This high frequency is mainly attributed to two factors: the inherent bias of TCRs generated by an otherwise random gene rearrangement process toward MHC recognition (6-8), and the high degree of cross-reactivity among $\mathrm{T}$ cells, also known as heterologous immunity $(9,10)$. Cross-reactivity occurs when a TCR specific to a microbial or other non-self peptide, bound to a self MHC molecule, also recognizes non-self MHC-peptide complexes (11).

The interaction between the TCR and the peptide-MHC complex lies at the heart of allorecognition and has been elucidated at the molecular level using x-ray crystallography (11-13). Notably, the TCR interacts with amino acid residues in the peptide bound to the MHC groove

\section{Delated Article: https://doi.org/10.1172/JCl146771}

Conflict of interest: The authors have declared that no conflict of interest exists.

and amino acids in the MHC molecule regions that surround the groove (Figure 1B). Moreover, empty MHC molecules that lack peptides are rare because of their instability; they have extremely short half-lives. In indirect allorecognition, the peptide is derived from a non-self donor protein while the MHC molecule is that of the recipient and is self (Figure 1A). Therefore, the peptide cargo clearly plays an important role in determining which TCR binds. In direct allorecognition, the peptide is almost always self while the MHC molecule is non-self (Figure 1A), begging the question of whether selfpeptides have any role in determining the TCR specificity of directly alloreactive $\mathrm{T}$ cells. Three answers have been proposed in the literature (reviewed in ref. 14): (a) the TCR recognizes a non-self MHC molecule irrespective of the self-peptide present (peptide independence), (b) the TCR recognizes one of several self-peptides complexed with the same non-self MHC molecule (peptide degeneracy), or (c) the TCR recognizes a unique non-self MHCself-peptide complex (peptide dependence; refs. 2, 3, 15). Resolving the debate is not a trivial matter as it would shed insight into the fundamental biology of the alloimmune response and would provide the means to identify and track alloreactive $\mathrm{T}$ cells using MHC-peptide multimers.

\section{Self-peptide dependence of direct allorecognition}

In this issue of the JCI, Son et al. used an elegant mouse transplantation tolerance model to provide compelling in vivo evidence for peptide dependence of direct allorecognition (16). In this model, adenoviral transduction of an allogeneic MHC I molecule into recipient hepatocytes rendered the mice tolerant to subsequent skin grafts bearing the same allogeneic MHC molecule. The researchers used two genetic approaches to demonstrate that exclusion of the normal endogenous liver immunopeptidome (the liver self-peptide repertoire) from the allogeneic MHC I 
A

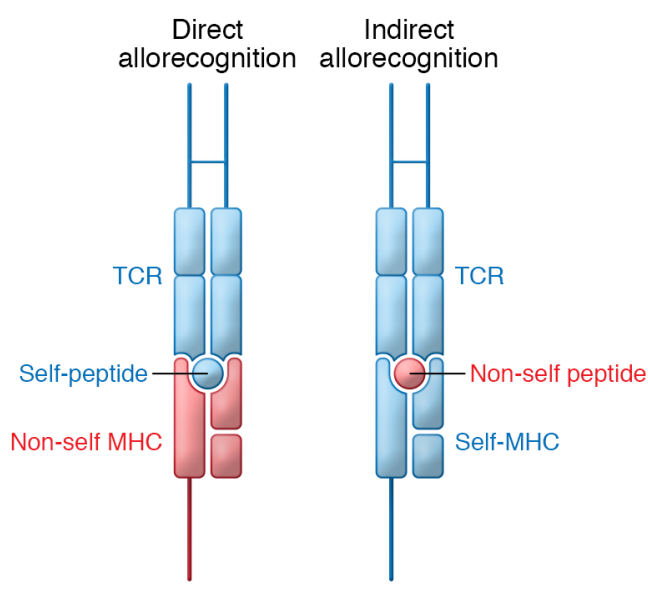

B

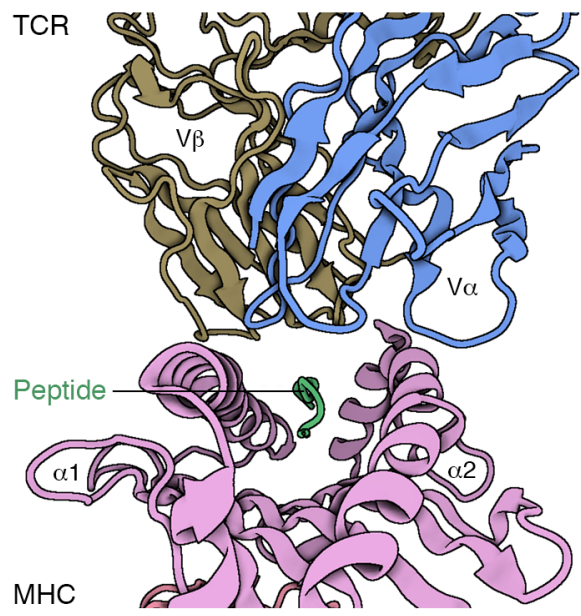

C

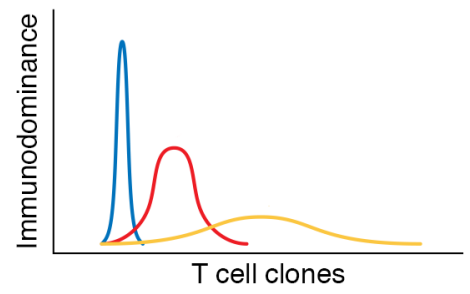

Figure 1. Peptide dependence of the alloimmune response. (A) Direct and indirect allorecognition is mediated by recipient TCRs. Direct allorecognition involves the binding of self (recipient) TCRs to non-self (donor) MHC molecules loaded with self-peptides, whereas indirect allorecognition is mediated by recipient TCRs that recognize non-self (donor) peptides bound to self (recipient) MHC molecules. (B) X-ray crystal structure binds the TCR and the peptideMHC complex. Note that the variable domains of the TCR, $V \alpha$ and $V \beta$, contact the peptide as well as the regions of the MHC molecule surrounding the peptide-binding groove. Figure created in Biorender. Protein Data Bank accession: 2CKB. (C) Direct T cell alloreactivity is the summation of many nondominant T cell clones (yellow), a small number of dominant T cell clones (blue), or an intermediate number of nondominant and dominant T cell clones (red). The experiments by Son et al. (16) suggest alloreactivity is the intermediate scenario (red).

molecule blocks tolerance induction. In the first approach, endogenous peptide presentation was excluded by transducing mice with a hepatocyte-specific adenoviral vector carrying a single-chain MHC I construct that restricted the peptide cargo to a defined single peptide. By occupying the MHC groove, the defined single peptide prevented naturally processed hepatocyte self-peptides from binding. This maneuver induced tolerance in alloreactive $\mathrm{T}$ cells bearing the TCR specific to the defined single peptide but prevented tolerance in the polyclonal alloreactive $\mathrm{T}$ cell population. In the second approach, the authors transduced mice that lacked hepatocyte expression of the transporter associated with antigen processing 1 (TAP1) protein with an adenoviral vector encoding a stable form of the allogeneic MHC I molecule (16). Since TAP1 is essential for loading endogenous cytosolic peptides onto MHC I molecules (17), TAP1 deficiency vastly reduced and altered the self-peptide repertoire bound to the allogeneic MHC I molecule. Tolerance to allografts transplanted from TAP1-sufficient donors bearing the allogeneic MHC I molecule was completely abrogated (16). Together, the two approaches established that induction of allospecific tolerance to a non-self $\mathrm{MHC}$ I molecule in vivo is critically dependent on the endogenous self-peptides bound to it. This conclusion applies equally well to the elicitation of rejection, as tolerance and rejection both depend on TCR recognition of alloantigen by the same alloreactive $\mathrm{T}$ cell pool (18). Therefore, direct alloreactivity, or to use more precise language, the TCR specificity of directly alloreactive $\mathrm{T}$ cells, is critically dependent on the self-peptides presented by the allogeneic MHC molecule.

\section{Tools to track polyclonal alloreactive T cells}

A critical gap in the field of transplantation immunology is the unavailability of tools to track polyclonal alloreactive $\mathrm{T}$ cells. Son and colleagues therefore set out to identify the self-peptides involved in direct $\mathrm{T}$ cell allorecognition of two allogeneic murine $\mathrm{MHC}$ I molecules, $\mathrm{H}-2 \mathrm{~K}^{\mathrm{b}}$ and $\mathrm{K}^{\mathrm{d}}$. Since allogeneic MHC I expressed in hepatocytes induced tolerance to skin and splenocyte grafts, the authors surmised that the self-peptides involved must be common to all three tissues. Elution of MHC I-bound peptides from these tissues identified 1000 or so common peptides. A subset of 100 peptides was selected for screening by synthesizing peptide-MHC tetramers and testing their binding to $\mathrm{T}$ cells from allosensitized mice. The authors found that 17 peptides were highly immu- nogenic, each binding to more than $5 \%$ of recipient $\mathrm{CD} 8^{+} \mathrm{T}$ cells. Surprisingly, five of these peptides, used together in a panel of peptide-MHC tetramers, were sufficient to identify $40 \%$ of all alloreactive $\mathrm{T}$ cells. The peptide-MHC tetramer panel was subsequently used to track alloreactive $\mathrm{T}$ cells in tolerized mice, demonstrating that the $\mathrm{T}$ cells had acquired an exhausted phenotype. Therefore, in addition to establishing a key role for self-peptides in direct alloreactivity, Son et al. succeeded in putting the peptides to use by creating a tool that identifies a sizeable proportion of the alloreactive $\mathrm{T}$ cell repertoire in one fell swoop (16).

\section{Conclusions and implications}

The findings by Son et al. (16) should be welcome news to transplant immunologists, particularly those studying transplantation across MHC barriers. For a very long time, we have lacked a clear understanding of the molecular specificity of directly alloreactive $\mathrm{T}$ cells as well as the tools to track them. For instance, it is unclear whether the pool of alloreactive $\mathrm{T}$ cells represents a summation of many different $\mathrm{T}$ cell clones, each present at a very low individual frequency, or a small number of $\mathrm{T}$ cell clones, each present at high frequency. In other words, is alloreactivity a broad hump of thousands of alloreactive T cell clones (Fig- 
ure $1 \mathrm{C}$, yellow curve), or is it carried out by a sharp peak of immunodominant $\mathrm{T}$ cell clones akin to antiviral responses (Figure $1 \mathrm{C}$, blue curve)? The Son et al. manuscript paints an intermediate picture (Figure 1C, red curve), one that offers a reprieve from the first, the broad hump, and implies that a sizeable proportion of alloreactive $\mathrm{T}$ cells can be tracked using only a handful of peptide-MHC multimers. This finding should pave the way toward unequivocal analysis of directly alloreactive polyclonal $\mathrm{T}$ cells during either rejection or tolerance, rather than inferring their biology from the behavior of surrogate TCR-transgenic T cells or by using blunt tools, such as the mixed lymphocyte reaction (MLR). Manipulating peptideMHC constructs should also open avenues for investigating whether altering the peptide cargo of MHC molecules can redirect the $\mathrm{T}$ cell response from rejection toward tolerance (19). Finally, researchers can apply approaches analogous to those of Son et al. (16) to make accurate tracking of the human alloimmune response a clinical reality.

\section{Acknowledgments}

HAA is funded by the American Gastroenterological Association and the Pittsburgh Liver Research Center (NIH P3ODK1120531). FGL is funded by NIH grants R01-AI049466 and R01-AI099465.
Address correspondence to: Hossam A. Abdelsamed, 200 Lothrop Street, W1555 Biomedical Science Tower, Pittsburgh, Pennsylvania 15261, USA. Phone: 412.383.5465; Email: abdelsamedha@ upmc.edu. Or to: Fadi G. Lakkis, 200 Lothrop Street, W1548 Biomedical Science Tower, Pittsburgh, Pennsylvania 15261, USA. Phone: 412.383.5774; Email: lakkisf@upmc.edu.

1. Lakkis FG, Lechler RI. Origin and biology of the allogeneic response. Cold Spring Harb Perspect Med. 2013;3(8):a014993.

2. Felix NJ, et al. Alloreactive T cells respond specifically to multiple distinct peptide-MHC complexes. Nat Immunol. 2007;8(4):388-397.

3. Morris GP, et al. Alloreactivity is limited by the endogenous peptide repertoire. Proc Natl Acad Sci US A. 2011;108(9):3695-3700.

4. Suchin EJ, et al. Quantifying the frequency of alloreactive T cells in vivo: new answers to an old question. J Immunol. 2001;166(2):973-981.

5 . Macedo C, et al. Contribution of naive and memory T-cell populations to the human alloimmune response. Am J Transplant. 2009;9(9):2057-2066.

6. Blackman $\mathrm{M}$, et al. The $\mathrm{T}$ cell repertoire may be biased in favor of $\mathrm{MHC}$ recognition. Cell. 1986;47(3):349-357.

7. Zerrahn J, et al. The MHC reactivity of the T cell repertoire prior to positive and negative selection. Cell. 1997;88(5):627-636.

8. Yin L, et al. T cells and their eons-old obsession with MHC. Immunol Rev. 2012;250(1):49-60.

9. Burrows S, et al. An alloresponse in humans is dominated by cytotoxic T lymphocytes (CTL) cross-reactive with a single Epstein-Barr virus CTL epitope: implications for graft-versus-host disease. J Exp Med. 1994;179:1155-1161.

10. Adams AB, et al. Heterologous immunity provides a potent barrier to transplantation tolerance. J Clin Invest. 2003;111(12):1887-1895.

11. Macdonald WA, et al. T cell allorecognition via molecular mimicry. Immunity. 2009;31(6):897-908.

12. Colf LA, et al. How a single T cell receptor recognizes both self and foreign MHC. Cell. 2007;129(1):135-146.

13. Varani L, et al. Solution mapping of T cell receptor docking footprints on peptide-MHC. Proc Natl Acad Sci U S A. 2007;104(32):13080-13085.

14. D'Orsogna LJ, et al. Endogenous-peptidedependent alloreactivity: new scientific insights and clinical implications. Tissue Antigens. 2013;81(6):399-407.

15. Amir AL, et al. Identification of a coordinated $\mathrm{CD} 8$ and CD4 $\mathrm{T}$ cell response directed against mismatched HLA Class I causing severe acute graft-versus-host disease. Biol Blood Marrow Transplant. 2012;18(2):210-219.

16. Son ET, et al. The self-peptide repertoire plays a critical role in transplant tolerance induction. J Clin Invest. 2021;131:e146771.

17. Neefjes JJ, et al. Selective and ATP-dependent translocation of peptides by the MHC-encoded transporter. Science. 1993;261(5122):769-771.

18. Madsen JC, et al. Immunological unresponsiveness induced by recipient cells transfected with donor MHC genes. Nature. 1988;332(6160):161-164.

19. Sloan-Lancaster J, et al. Induction of T-cell anergy by altered T-cell-receptor ligand on live antigen-presenting cells. Nature. 1993;363(6425):156-159. 